\title{
GOÄ-Tipp: Keine Ziffer bei der Venenbehandlung vergessen
}

\section{Hier steht eine Anzeige.}

Eine Varikosis oder gar ein postthrombotisches Syndrom der unteren Extremitäten ist bei den betroffenen $\mathrm{Pa}$ tienten oft mit weitreichenden Folgeerkrankungen verbunden. Die venöse Stauung hat einen negativen Einfluss auf die Hautdurchblutung, es kommt zu einer Stauungsdermatose bzw. -dermatitis oder auch zu einem Ulcus cruris. Im Gegensatz zur möglichen Leistungsabrechnung im EBM bietet sich in der GOÄ dabei eine sehr differenzierte Vorgehensweise an - der Arzt hat also bessere Möglichkeiten, seine tatsächliche Leistung abzubilden.

\section{MMW-KOMMENTAR}

Leider muss der behandelnde Arzt eine Vielzahl von Ausschlussbestimmungen, Kommentierungen und sogar Gerichtsurteile beachten, wenn er seine Leistungen bei Patien-

\section{Tab. 1 Abrechnungsbeispiel: Patienten mit Ulcus cruris bei postthrombotischem Syndrom und Stauungsdermatitis beidseitig}

\begin{tabular}{|c|c|c|c|}
\hline GOÄ & Legende & Faktor & Euro \\
\hline 1 & Beratung & 2,3 & 10,71 \\
\hline 5 & $\begin{array}{l}\text { Einfache } \\
\text { Untersuchung }\end{array}$ & 2,3 & 10,71 \\
\hline 644 & $\begin{array}{l}\text { Direktionaler } \\
\text { Doppler }\end{array}$ & 1,8 & 18,88 \\
\hline 2006 & $\begin{array}{l}\text { Behandlung } \\
\text { Ulcus cruris }\end{array}$ & 2,3 & 8,44 \\
\hline 209 & $\begin{array}{l}\text { Salbenauftragung } \\
\text { bei Stauungs- } \\
\text { dermatitis }\end{array}$ & 2,3 & 20,10 \\
\hline 200 & Abdeckverband & 2,3 & 6,02 \\
\hline 203 & $\begin{array}{l}\text { Kompressions- } \\
\text { verband beidseitig }\end{array}$ & 2,3 & 25,48 \\
\hline \multicolumn{3}{|c|}{ Summe: } & 100,34 \\
\hline
\end{tabular}

ten mit einem solchen Krankheitsbild korrekt nach GOÄ abrechnen möchte. Die hier normalerweise nötigen Eingangsberatungen und Untersuchungen können nach den Nrn. 1 und 5 berechnet werden - in Verbindung mit den notwendigen Sonderleistungen aber nur einmal im Behandlungsfall. Zur Erinnerung: Dieser ist in der GOÄ anders als im EBM nicht als Quartal definiert, sondern als der Zeitraum von einem Monat ab Behandlungsbeginn.

Bei der notwendigen Lokalbehandlung geht es dann mit den Abrechnungsregelungen weiter. Üblicherweise können bei operativen Leistungen wie der Versorgung eines Ulcus cruris korrespondierende Verbände nicht berechnet werden, oder es können verschiedene Verbandformen nicht kombiniert werden. Bei der Behandlung von venösen Stauungen und ihren Folgen ist das allerdings anders. Schon 1968 bestimmte das Landessozialgericht Nordrhein-Westfalen, dass unterschiedliche Verbände im Zusammenhang mit der Versorgung desselben Bereichs der Körperoberfläche durchaus nebeneinander berechnungsfähig sind. Bedingung dafür ist allerdings, dass die Anwendung zweier unterschiedlicher therapeutischer Wirkprinzipien auch tatsächlich medizinisch notwendig ist (Az.: L1 Ka 34/68). So kann beispielsweise im Zusammenhang mit der Behandlung eines Ulcus cruris neben dem unmittelbar auf das Ulkus wirkenden Verband (Nr. 200 GOÄ) ein zusätzlicher Kompressionsverband des betroffenen Beines (Nr. 204) berechnet werden.

Beachtenswert ist auch eine weitere Regelung zu Verbänden. In den Leistungen nach den Nrn. 2000-2005 ist der Verband nach Nr. 200 enthalten - nicht aber in der Behandlung einer sekundär heilenden Wunde nach Nr. 2006. Dies geht auf eine Entscheidung der Bundesärztekammer vom 30. August 1991 zurück. 